\title{
Circuit Theory Analysis of Parity-Time-symmetric Wireless Power Transfer System
}

\author{
Yu Jin, Wangqiang Niu, Wei Gu \\ Key Laboratory of Transport Industry of Marine Technology and Control Engineering, \\ Shanghai Maritime University, Shanghai 201306, China
}

\author{
Received: April 16, 2020. Revised: June 11, 2020. 2nd Revised: June 17, 2020. \\ Accepted: June 18, 2020. Published: June 19, 2020.
}

\begin{abstract}
Wireless power transfer (WPT) technology reduces the risks brought by connection between electrical equipment and power source, it has been widely used in various fields in recent years. To overcome issues of low transfer efficiency and poor robustness when coupling coefficient varies, a WPT system based on parity-time (PT) symmetric circuit is proposed, which consists of two RLC oscillators. The system state equation is obtained by circuit theory, then be analyzed to derive the system resonance frequency, transmission efficiency, and phase difference between $\mathrm{Tx}$ and Rx. A simulation based on PSIM is established to verify the theoretical derivation of transmission characteristics. The simulation results illustrate that the resonance frequency of the WPT system is adjusted automatically in the strong coupling region when the coupling coefficient changes, the output voltage across the load resistance always equals source voltage on the transmitter. Compared with the non-parity-time symmetric system, PT-symmetric WPT system could achieve higher transfer efficiency over a longer distance, this scheme can transfer power with constant efficiency of over $80 \%$ in a certain region. A set of simulations with variation load resistance are considered to verify the system robustness. All results are consistent with theoretical derivation and analysis.
\end{abstract}

Keywords -Wireless power transfer; Parity-time-symmetry; Circuit model; Power transmission characteristics.

\section{INTRODUCTION}

$\mathrm{W}$ IRELESS power transfer (WPT) technology has been extensively used in various occasions, such as mobile devices and electric vehicles, autonomous underwater vehicles (AUV) and automated guided vehicles (AGV). It is greatly improving the flexibility of electrical equipment, makes the repair of the faulty device easier. However, low transfer efficiency and poor robustness are two main issues of the
WPT system [1], [2]. To implement continuous and efficient power transmission, four common types of WPT technology are introduced: magnetic resonance WPT (MRWPT), inductive WPT (IWPT), microwave WPT (MWPT) and laser WPT (LWPT). Nevertheless, the IWPT system may have an adverse effect on people or things in two coils; the transfer efficiency of MWPT is relatively low [3]; the cost of LWPT is much more than other methods. So researches more focus on the MRWPT method [4]-[7], which has a strong anti-interference ability to the surrounding environment. The phenomenon of frequency bifurcation appears in the MRWPT system in the strong coupling region, resonance frequency of system varies when the variation of coupling coefficient, the operating frequency of the system does not adjust automatically with the resonance frequency, which causing a constantly changing transfer efficiency.. This phenomenon was demonstrated in [8] and concluded that the maximum load power could be obtained at the frequency bifurcation point. Reference [9] established a circuit model and made an exact explanation for the frequency bifurcation phenomenon. The further analysis of contactless power transfer characteristics with relay coils was studied in [10] using coupled mode theory and practical experience, which concluded that the type of frequency bifurcation changes with the number of relay coils. To obtain higher and constantly transfer efficiency, the operating frequency of WPT system should track the resonant frequency.

References [11]-[14] analyzed the output power characteristics of WPT system to obtained optimal solutions, then a negative feedback control module was designed to track specific parameters at the transmitter or receiver, such as the mean value of current sampling on the transmitter at the voltage commutation point [15]. Frequency tracking detuning control strategy based on the differential link phase-locked loop was also commonly used to implement constant power transfer [16]. References [17] and [18] offered reactive power compensation for contactless power transfer systems with variable load. Reference [19] pointed out a situation that 
non-resonance maximizes the efficiency of power transmission, considering both output power and efficiency, then decide a balanced working frequency under the condition of resonance is different with the maximal efficiency. Feedback mechanisms and compensation mechanisms were utilized to achieve efficient power transmission. The shape and structure of WPT system also affect transfer efficiency. A novel meander-type laminated coupled structure was proposed in [20], with higher transmission efficiency and output power. The above researches achieved efficient power transmission by utilizing an additional feedback loop, but the poor real-time performance and high cost were also should be regarded.

The PT-symmetry principle was originated from quantum physics. The first experiment of PT-symmetric circuit was performed by Professor J Schindler of Wesleyan University in 2011, the experimental circuit structure consisted of a pair of coupled LRC oscillators. The transmitter contained a nonlinear gain whereas the receiver had the same amount of attenuation, which offered the simplest implementation of a PT-symmetry system in the electronic field [21]. Then Professor J Schindler established PT-symmetric dimer modes to express all universal phenomena encountered in systems with generalized PT-symmetric in the next year [22]. In 2017, Professor Fan of Stanford University applied PT-symmetric circuit to WPT system [23] and successfully lighted up a $60 \mathrm{~W}$ lamp in 2 meters, achieved robust against variations in distance. Reference [26] proposed a new dual-coupled robust WPT model based on the PT-symmetry principle of [24] to implement efficient and stable power transmission. Considering the applications of PT-symmetry WPT system, [25] and [27] applied PT-symmetric circuits to charge fuzes with variable gap and wireless charging for drone systems, respectively. A research on the transmitter amplification of negative resistance based on PT-symmetry principle was presented in [28], that summed up the amplification was adjusted automatically with varies distance. An ultra-sensitive sensor based on parity-inversion and time-inversion was proposed in [29], which could detect changes of resistance or reactance accurately in a circuit. References [30], [31] analyzed the PT-symmetric circuits and transmission characteristics of WPT systems from the perspective of the Duffing inequality, it also opened a new direction toward investigating novel analytical method for the PT symmetrical quantum system in WPT technology.

Coupled mode theory and circuit theory are two main methods to analyze the WPT system. The coupled mode theory analyzed the WPT system from the perspective of energy, made solving process easier by wrote reduced-order state equations [9], [23], [27]. Nevertheless, the accuracy of solutions may reduce. Circuit theory model gained the system state equation based on Kirchhoff's voltage law and Kirchhoff's current law, the formulation of voltage and current of each node and branch were given, then transfer efficiency of WPT system was solved [14]-[16], [24], [25]. Furthermore, output voltage values of negative resistance and load resistance, phase difference characteristics are presented by circuit theory method. Reference [32] built several topologies of WPT system by coupled mode theory, the relationship between the energy model and coupling mode was discussed, the mathematical significance of their equivalence is studied, ultimately, simulations based on MATLAB were used to verify the theoretical analysis. Current and voltage are the main parameters in WPT system and easy to understand in the field of electronics, so circuit theory method is utilized in this paper. This paper analyzes the characteristics of WPT system based on PT-symmetric circuit by circuit theory method, which focuses on the amplitude, phase and waveform of output voltage on transmitter and receiver. Variation of transfer efficiency and operating angular frequency during resonance is also expressed in this paper, and simulations demonstrate the accuracy of theoretical model. A comparison between PT-symmetry WPT system and Non-PT (NPT) symmetry WPT system on the above characteristic is given, that verify stability and efficiency of PT-symmetry principle applied to WPT system. The remaining of this paper is organized as follows: Section II expresses the theory of PT-symmetry, circuit theory model, theoretical analysis of PT-symmetric characteristics in WPT system. Simulations based on PSIM verify theoretical derivations in Section III. Finally, Section IV concludes the paper.

\section{MATERIALS AND METHODS}

\section{A. Theory of Parity-Time-Symmetry}

The parity-time-symmetry is a property described by the invariance of parity-inversion and time-inversion. This theory has been used in optics, high energy physics and field theory, WPT system has familiar transmission principle with optical coupling application, that is the reason why PT-symmetric theory could be used in WPT system. Parity-inversion is defined by the inversion of space, in continuous space coordinates. Parity-inversion means mirroring from the space coordinates of $x \leftrightarrow-x$. Similarly, the odd powers to $x$ and their odd derivatives also have corresponding mirroring properties [13]. Time-inversion indicates the reversal of time direction, that is, $t \leftrightarrow-t$, the odd-numbered derivative of time $t$ also needs sign transformation. The PT-symmetric system is realized by a symmetric circuit model with gain element and loss element. When the gain and loss of the system are same value with opposite sign, the energy flows steadily on both sides of the system and then reach equilibrium. Nevertheless, this stable transmission state will be broken with the variation of a certain parameter, it is a spontaneous characteristic of the PT-symmetric system.

This paper studies the application of PT-symmetric circuit in WPT system from the aspect of electronics, parity symmetry can be simplified to the symmetry of circuit topology with same elements on both sides, such as series-series (S-S) RLC topology and parallel-parallel (P-P) RLC topology. Parity-inversion of the circuit system is achieved by interchanging the signs on both sides, that is, $x \leftrightarrow$ $-x, p \leftrightarrow-p$. Time-inversion is represented by swapping the sign of the purely resistive impedance whereas keeping the sign of reactance part in the circuit, that is $t \leftrightarrow-t, p \leftrightarrow-p$. 
Assuming $H$ indicates a system described by a Hamiltonian under the following condition, then this system is a PT-symmetric system. Combined with the circuit analysis theory, system state equation unchanged when swapping the time sign and state variable.

$$
\left\{\begin{array}{c}
{[H, P T]=0} \\
{[H, P] \neq 0} \\
{[H, T] \neq 0}
\end{array}\right.
$$

From the perspective of wireless power transfer, if the output power at the receiver remains unchanged, means the WPT system could meet PT-symmetry within a certain range, which greatly improves the stability of transmission and implements robust power transfer. Part $B$ and $C$ demonstrate the property of PT-symmetry of the proposed WPT system.

\section{B. Circuit model of WPT system}

The PT-symmetric circuit is composed of two RLC oscillators as an S-S topology (as shown in Fig. 1). The difference between these two oscillators is that the non-linear gain on the transmitter is consists of a negative resistance, which contains an operational amplifier and a resistor. In the PT-symmetric circuit model, $L_{T}$ and $L_{R}$ are the inductance of Tx and Rx, $C_{T}$ and $C_{R}$ present the capacitors in transmitter and receiver. $R_{T}$ and $R_{R}$ presents the internal loss of Tx and Rx. $M$ is the mutual inductance between the two coils, $R_{L}$ indicates load resistance at receiver, and $A$ is the amplification of the operational amplifier. $R_{0}$ is a series resistor on the transmitter, which constitutes a non-linear negative resistance together with an operational amplifier.

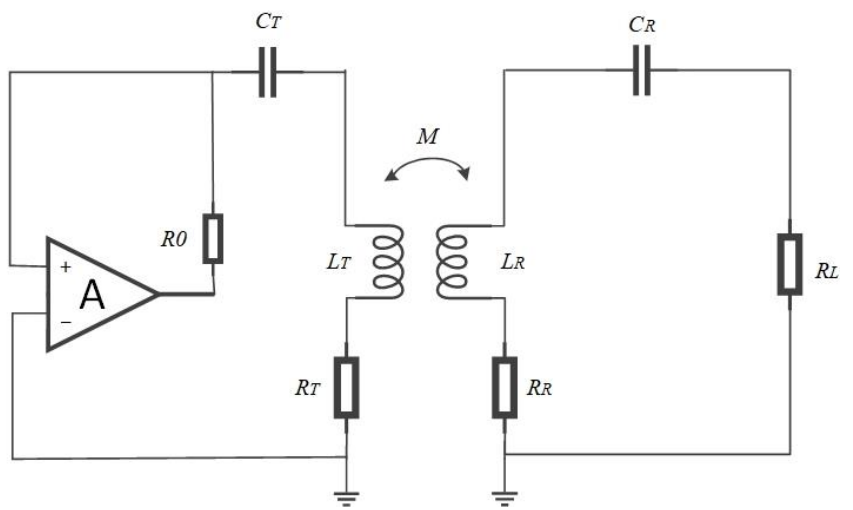

Fig. 1 Structure of WPT system based on PT-symmetric circuit

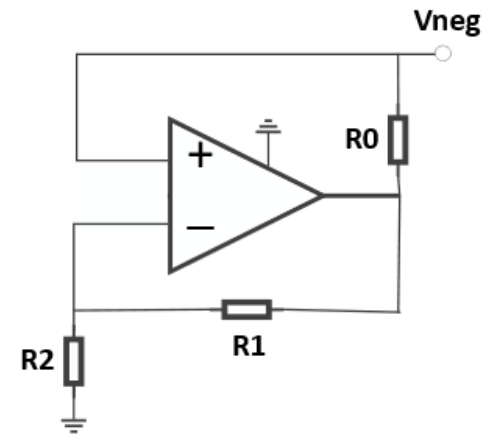

Fig. 2 Nonlinear negative resistance circuit

The nonlinear negative resistance circuit is illustrated in
Fig. 2. $V_{\text {neg }}$ represents the voltage across the nonlinear gain module, the amplification of operational amplifier $A=\frac{R_{1}+R_{2}}{R_{2}}$, the value of equivalent negative resistance $|-R|=\frac{R_{2} R_{0}}{R_{1}}=\frac{R_{0}}{A-1}$. The direction of output voltage $V_{\text {neg }}$ is opposite to the current on the transmitter, and the nonlinear negative resistance is an active component, which is the key of the stable power transmission.

An equivalent circuit model of the PT-symmetric wireless power transmission system is shown in Fig. 3.

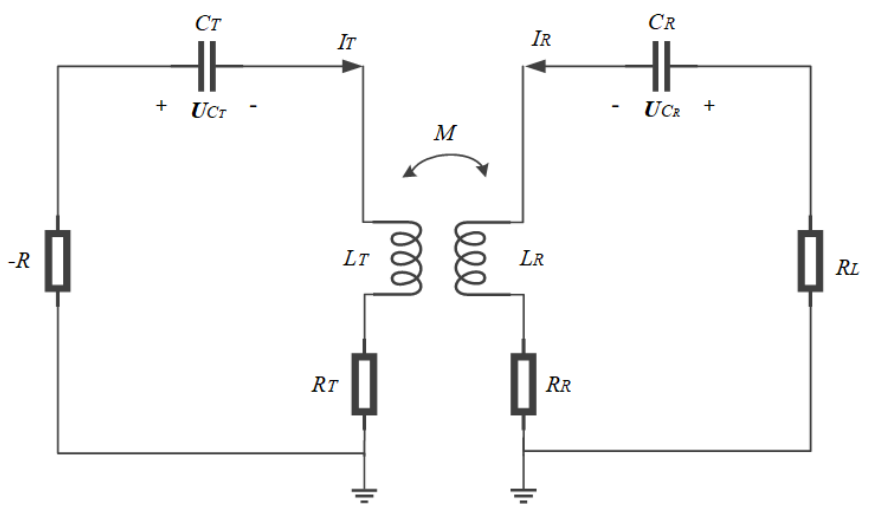

Fig. 3 Equivalent circuit model of PT-symmetric WPT system

The parameters of transmitter and receiver are designed to the same with the purpose of meet the condition of PT-symmetry. In detail, $|-R|=R_{L}=R, L_{T}=L_{R}=L, C_{T}=C_{R}=$ $C, R_{T}=R_{R}$. The corresponding circuit equation is obtained by Kirchhoff's law of voltage and current:

$$
\left\{\begin{array}{c}
\dot{I}_{T}\left(R_{T}-R\right)+\dot{U}_{C_{T}}+j w_{1} L_{T} \dot{I}_{T}+j w_{1} M \dot{I}_{R}=0 \\
\dot{I}_{R}\left(R_{L}+R_{R}\right)+\dot{U}_{C_{R}}+j w_{1} M \dot{I}_{T}+j w_{1} L_{R} \dot{I}_{R}=0 \\
\dot{I}_{T}=j w_{1} C_{T} \dot{U}_{C_{T}} \\
I_{R}=j w_{1} C_{R} \dot{U}_{C_{R}}
\end{array}\right.
$$

where $w_{1}$ is the operating angular frequency of the RLC resonant system, wo indicates the natural angular frequency. Substituting the voltage parameters in (2), an equation about $\dot{I}_{T}$ and $\dot{I}_{R}$ is obtained:

$\left[\begin{array}{cc}-j \gamma+\frac{1}{w\left(1-k^{2}\right)}-w & -\frac{k}{w\left(1-k^{2}\right)} \\ -\frac{k}{w\left(1-k^{2}\right)} & j \gamma+\frac{1}{w\left(1-k^{2}\right)}-w\end{array}\right]\left[\begin{array}{l}\dot{I}_{T} \\ \dot{I}_{R}\end{array}\right]=0$

where $\gamma=R \sqrt{\frac{C}{L}}, k=\frac{M}{L}, w=\frac{w_{1}}{w_{0}}$, the coefficient matrix of the current state equation is a symmetric matrix, and the circuit equation is unchanged after swapping the subscripts $T$, $R$ of current $I$, this is the preliminary proof of the PT-symmetry WPT system.

Let the determinant of the coefficient matrix equal 0 :

$$
\begin{gathered}
\left(\frac{1}{w^{2}\left(1-k^{2}\right)}-\frac{2}{1-k^{2}}+w^{2}-\gamma^{2}\right) \\
-j 2 \gamma\left(\frac{1}{w\left(1-k^{2}\right)}-w\right)=0
\end{gathered}
$$


the solutions of (3) are:

$$
\begin{aligned}
& w_{1,2}= \pm \frac{1}{2} w_{0}\left(\sqrt{\gamma_{u}^{2}-\gamma^{2}}+\sqrt{\gamma_{P T}^{2}-\gamma^{2}}\right) \\
& w_{3,4}= \pm \frac{1}{2} w_{0}\left(\sqrt{\gamma_{u}^{2}-\gamma^{2}}-\sqrt{\gamma_{P T}^{2}-\gamma^{2}}\right)
\end{aligned}
$$

where $\gamma_{u}$ presents the upper critical point of the system, and $\gamma_{P T}$ means the PT symmetry breaking point of the system.

The relationship with the coupling coefficient is illustrated in (6) and (7). The working range of WPT system is divided into three parts by $\gamma_{u}, \gamma_{P T}$.

$$
\begin{aligned}
\gamma_{u} & =\frac{1}{\sqrt{1-k}}+\frac{1}{\sqrt{1+k}} \\
\gamma_{P T} & =\frac{1}{\sqrt{1-k}}-\frac{1}{\sqrt{1+k}}
\end{aligned}
$$

When $0<\gamma<\gamma_{P T}$, the eigenvalues of the system are four reals, which contain two pairs of numbers with opposite signs. This region is PT symmetric region, the WPT system performs equal-amplitude oscillation, power and voltage keep equal on the transmitter and receiver. From the perspective of wireless power transfer, it also called strong coupling region [13]. The resonance frequency and phase difference between transmitter and receiver change continuously with the coupling coefficient in the strong coupling region. To analysis the dynamic characteristics of PT symmetric circuit quantitatively, let the phase $\emptyset=\emptyset_{T}-\emptyset_{R}$, where $\emptyset_{T}$ presents the phase on transmitter and $\emptyset_{R}$ correspond to the receiver. $\emptyset_{h}$ and $\emptyset_{l}$ indicates the phase difference on the low-frequency branch and high-frequency branch in the strong coupling region, respectively. The phase difference is $\{0, \pi\}$ when $\gamma=0$, the phase difference of the high-frequency branch increases from 0 and the low-frequency branch decreases from $\pi$, as shown in (8):

$$
\begin{gathered}
\emptyset_{l}=\pi-\tan ^{-1}\left[\frac{1}{\gamma}\left(\frac{1}{\left(1-k^{2}\right)^{-3 / 4}}-\left(1-k^{2}\right)^{-1 / 4}\right)\right] \\
\emptyset_{h}=\tan ^{-1}\left[\frac{1}{\gamma}\left(\frac{1}{\left(1-k^{2}\right)^{-3 / 4}}-\left(1-k^{2}\right)^{-1 / 4}\right)\right]
\end{gathered}
$$

When $\gamma_{P T}<\gamma<\gamma_{u}$, the eigenfrequencies of the system become two pairs of conjugate complex numbers with non-zero real parts. This region is PT symmetric broken region, also known as weak coupling region. The WPT system does not meet PT-symmetry anymore in this range, an exponential attenuation mode and an exponentially increasing mode occur simultaneously, these two modes oscillate at the same frequency. The phase difference between transmitter and receiver does not change much.

When $\gamma>\gamma_{P T}$, the phase difference is $\emptyset=\emptyset_{\gamma_{P T}}$, where $\emptyset_{\gamma_{P T}}$ is the phase difference that corresponds to $\gamma=\gamma_{P T}$. The eigenfrequency of the system becomes two pairs of pure imaginary numbers. There is no oscillation mode in the system, which reflects the over-damped state in the RLC oscillator circuit.

\section{PT-symmetry in WPT system}

To verify the property of PT-symmetry in the WPT system furtherly, the capacitor voltages of the transmitter and the receiver are taken as state variables, (9) is the system state equation based on $U_{C_{T}}$ and $U_{C_{R}}$ :

$$
\left\{\begin{array}{c}
C_{T} \frac{L_{T} L_{R}-M^{2}}{L_{R}} \frac{d^{2} U_{C_{T}}}{d t^{2}}+\left(R+R_{T}\right) C_{T} \frac{d U_{C_{T}}}{d t}+U_{C_{T}} \\
-\frac{M R_{L} C_{R}}{L_{R}} \frac{d U_{C_{R}}}{d t}-\frac{M}{L_{R}} U_{C_{R}}=0 \\
C_{R} \frac{L_{T} L_{R}-M^{2}}{L_{T}} \frac{d^{2} U_{C_{R}}}{d t^{2}}+\left(R_{L}+R_{R}\right) C_{R} \frac{d U_{C_{R}}}{d t}+U_{C_{R}} \\
-\frac{M R_{T} C_{T}}{L_{T}} \frac{d U_{C_{T}}}{d t}-\frac{M}{L_{T}} U_{C_{T}}=0
\end{array}\right.
$$

The state equation of the system keeps the same after the parity-inversion and time-inversion, and the voltages at the transmitter and the receiver oscillate with the same amplitude. Therefore, S-S topology RLC oscillators meet the property of PT-symmetry could be used as a PT-symmetric circuit model. Considering the power transfer between transmitter and receiver, let $q_{C_{T}}=C_{T} U_{c_{T}}, q_{C_{R}}=C_{R} U_{c_{R}}$, based on (9), a system state equation about the capacitance in capacitors at transmitter and receiver is obtained, as presents follow:

$$
\left\{\begin{aligned}
\frac{d^{2} q_{C_{T}}}{d t^{2}} & =-\frac{\left(R+R_{T}\right) L_{R}}{L_{T} L_{R}-M^{2}} \frac{d q_{C_{T}}}{d t}-\frac{L_{R}}{C_{T}} \frac{1}{L_{T} L_{R}-M^{2}} q_{C_{T}} \\
& +\frac{M R_{L}}{L_{T} L_{R}-M^{2}} \frac{d q_{C_{R}}}{d t}+\frac{M}{C_{R}} \frac{1}{L_{T} L_{R}-M^{2}} q_{C_{R}} \\
\frac{d^{2} q_{C_{R}}}{d t^{2}} & =-\frac{\left(R_{L}+R_{R}\right) L_{T}}{L_{T} L_{R}-M^{2}} \frac{d q_{C_{R}}}{d t}-\frac{L_{T}}{C_{R}} \frac{1}{L_{T} L_{R}-M^{2}} q_{C_{R}} \\
+ & \frac{M R}{L_{T} L_{R}-M^{2}} \frac{d q_{C_{T}}}{d t}+\frac{M}{C_{T}} \frac{1}{L_{T} L_{R}-M^{2}} q_{C_{T}}
\end{aligned}\right.
$$

Similarly, swapping the subscript of capacitors at both sides for parity-inversion, and interchange $t$ with $-t$ for time-inversion, the same equation as the original equation is obtained, that is, the system transmission characteristics and charges on transmitter and receiver maintain invariable during parity-time inversion.

\section{Analysis of transmission characteristics}

Negative resistance offers power for the WPT system as an active component in the transmitter. $I_{R}$ and $V_{\text {load }}$ have the same phase, whereas $I_{T}$ and $V_{\text {neg }}$ are the opposite. The transfer efficiency is calculated by the ratio of active power between transmitter and receiver, so the transfer efficiency of the PT-symmetric WPT system is defined as load power divided by the output power of nonlinear negative resistance, as follows:

$$
\eta=\frac{P_{\text {load }}}{\left|P_{\text {neg }}\right|}=\frac{I_{R} V_{\text {load }}}{\left|I_{T} V_{\text {neg }}\right|}
$$

Let the coupling rate of the WPT system presents by: 


$$
K=\frac{k w_{0}}{2}
$$

$R_{10}$ and $R_{20}$ indicate the loss rates of transmitter and receiver, respectively.

$$
\begin{gathered}
R_{10}=\frac{R_{T}}{2 L_{T}} \\
R_{20}=\frac{R_{R}+R_{L}}{2 L_{R}}
\end{gathered}
$$

The transfer efficiency of the WPT system can be calculated by:

$$
\eta= \begin{cases}\frac{R_{L}}{R_{T}+R_{R}+R_{L}} & \left(\gamma<\gamma_{P T}\right) \\ \frac{1}{1+\frac{R_{10} R_{20}}{K^{2}}} & \left(\gamma>\gamma_{P T}\right)\end{cases}
$$

In terms of the coupling coefficient $k$, the PT symmetric range (strong coupling region) is under the condition of $k>$ $k_{P T}$, the receiver is in a resonance state with an equal amplitude of transmitter, the transfer efficiency is close to $100 \%$ in this region, and the transfer efficiency is independent of the coupling coefficient between Tx and Rx. The PT symmetric broken range (weak coupling region) is under the condition of $k<k_{P T}$, the voltage at the receiver is not exactly equal to the output voltage of nonlinear negative resistance. The transfer efficiency of the system is related to the coupling coefficient between the two coils and gradually reduces as the coupling coefficient decreases.

\section{RESULTS}

Based on the PT-symmetry principle and circuit theory method, a simulation of the WPT system is performed by PSIM. The output voltage of nonlinear negative resistance and load resistance is measured to infer PT-symmetry. The parameters of the simulation are shown in Table 1. A small voltage on the transmitter capacitor starts the oscillation. Circuit parameters on the transmitter and receiver are approximately equal to meet PT-symmetry principle. The mutual inductance varies from $3.6 \mathrm{uH}$ to $31.2 \mathrm{uH}$, corresponding coupling coefficient at the range of 0.03-0.24.

Table 1 Parameters of WPT system

\begin{tabular}{c|c|c}
\hline Parameters & Tx & $\mathrm{Rx}$ \\
\hline Inductance $\left(\mathrm{L}_{1,2}\right)$ & $120 \mathrm{uH}$ & $120 \mathrm{uH}$ \\
\hline Capacitance $\left(\mathrm{C}_{1,2}\right)$ & $44 \mathrm{pF}$ & $45 \mathrm{pF}(43 \mathrm{pF})$ \\
\hline Resonant frequency (f0) & $2.19 \mathrm{MHz}$ & $2.19 \mathrm{MHz}$ \\
\hline Amplification $(\mathrm{A})$ & \multicolumn{2}{|c}{1.033} \\
\hline Mutual inductance & \multicolumn{2}{|c}{$3.6-31.2 \mathrm{uH}$} \\
\hline Gain resistance $\left(\mathrm{R}_{0}\right)$ & \multicolumn{2}{|c}{$5 \Omega$} \\
\hline Load resistance $\left(\mathrm{R}_{\mathrm{L}}\right)$ & \multicolumn{2}{|c}{$150 \Omega$} \\
\hline
\end{tabular}

Fig. 4 presents the relationship between the coupling coefficient and system resonant frequency. As the coupling coefficient decreases, the system resonances frequency varies from $1.97 \mathrm{MHz}$ to $2.52 \mathrm{MHz}$ by tracing two different frequency branches, then these two frequency branches converge at a critical coupling coefficient between strong coupled region and weak coupled region, that is, $k=0.0903$. Then system operating frequency stay at natural resonance frequency.

Fig. 4 Frequency bifurcation characteristic of WPT system

In the case of $k>0.0903$, two frequency branches appear with the coupling coefficient increases, the difference between high-frequency branch and low-frequency branch decreases as the coupling coefficient reduces. Theoretical values of the resonance frequency are presented by the black line in Fig. 4, which correspond to formula (5). Two sets of simulation results of frequency bifurcation characteristic are indicated by red dots and blue dots, the first set of simulation tracking the high-frequency branch whereas reduces the capacitance by $2 \mathrm{pF}$, the second simulation performs a blue dot curve and tracks the low-frequency branch spontaneously. It is found that frequency bifurcation tracking mechanism of the PTsymmetric system is related to the series capacitance of the transmitter and receiver, the system resonance frequency follows the high-frequency branch when $\mathrm{C} 1$ greater than $\mathrm{C} 2$ whereas tracking low-frequency branch when $\mathrm{C} 1$ less than $\mathrm{C} 2$.

In the case of $k<0.0903$, the WPT system is in weak coupling region, and the natural resonant frequency of the system does not change anymore. The simulation results are in good agreement with the theoretical derivation, which demonstrated the PT-symmetry WPT system has the ability to adjust the resonance frequency with varies coupling coefficient automatically.

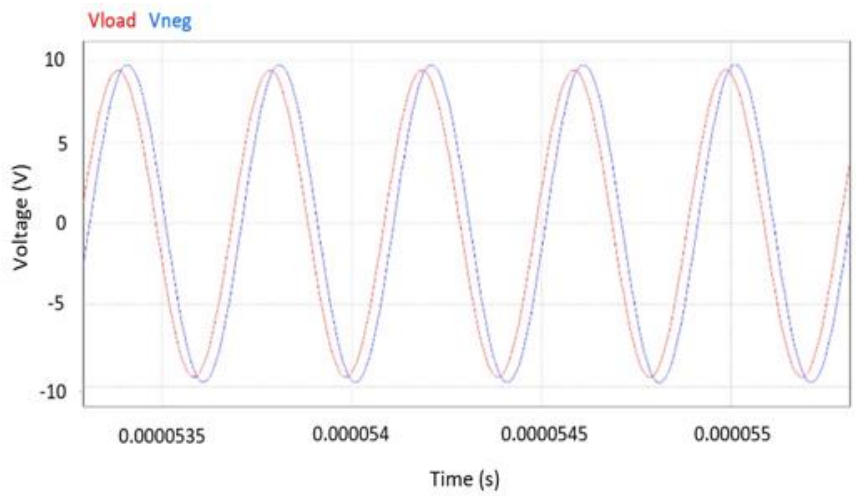

Fig. 5 Voltage waveform of negative resistance and load resistance while tracking high-frequency branch

Fig. 5 and Fig. 6 indicate the waveforms of the nonlinear negative resistance output voltage (blue line) and the load resistance output voltage(red line) when the system resonance frequency follows high-frequency branch and low-frequency branch. Under the condition of $k=0.2$, the system resonance frequencies are $2.4 \mathrm{MHz}$ and $2.03 \mathrm{MHz}$, respectively. The voltage value of load resistance and nonlinear negative resistance is approximately equal, these results consistent with the explanation in Section II, System energy flows steadily on transmitter and receiver. The phase difference approach to 0 
when tracking high-frequency, whereas $\pi$ is presented while tracking low-frequency, which coincidence with the theoretical derivation in section III.

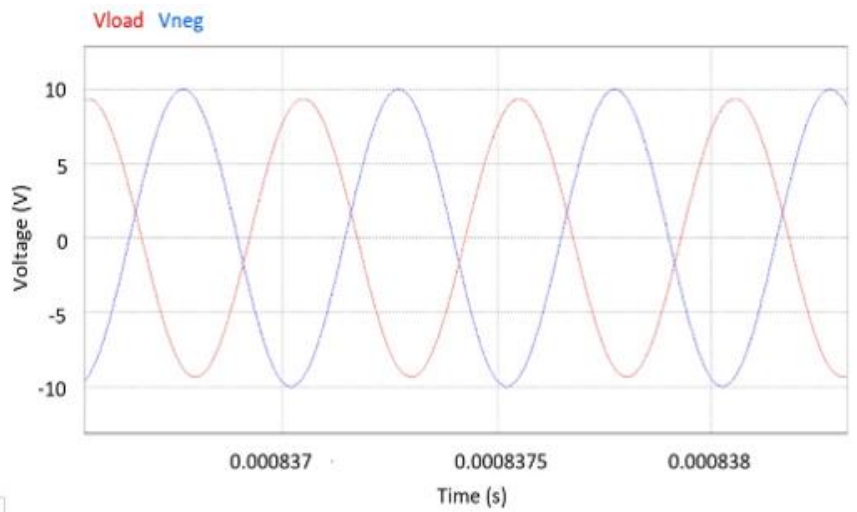

Fig. 6 Voltage waveform of negative resistance and load resistance while tracking low-frequency branch

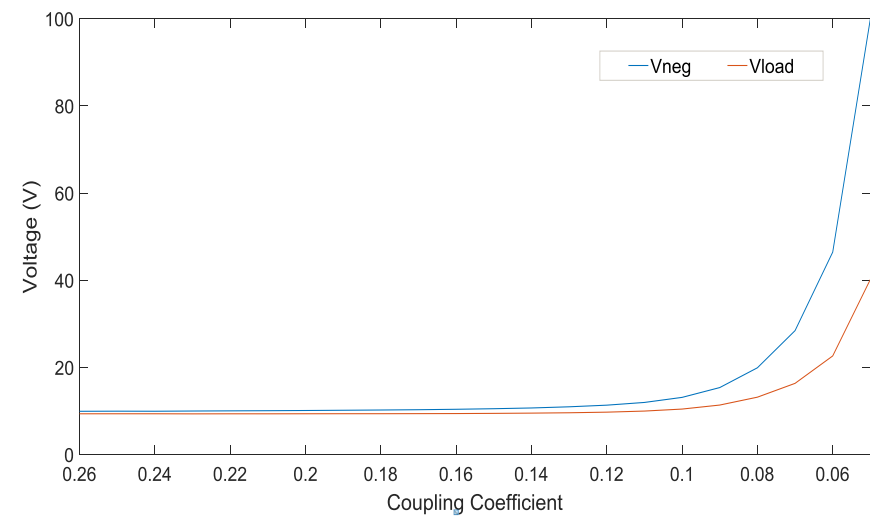

Fig. 7 Output valtage value of negative resistance and load resistance

The variation of nonlinear negative resistance voltage $V_{n e g}$ (blue line) and load voltage $V_{\text {load }}$ (red line) with the coupling coefficient is illustrated in Fig. 7. The voltages both transmitter and receiver are maintained at $10 \mathrm{~V}$ in the strong coupling region, which meets robustness.

The phase difference between the output voltage of the nonlinear negative resistance and the load resistance voltage accord with the theoretical value (see Fig. 8), black line indicates the derivation results of equation (8). When the system resonance frequency tracks the high-frequency branch (red line), the phase difference gradually increases from 0 whereas tracking the low-frequency branch (blue line) decreases from $\pi$. In the weak coupling region, both two branches are remains approximately $\frac{\pi}{2}$ and basically unchanged. The simulation results roughly agree with the derivations.

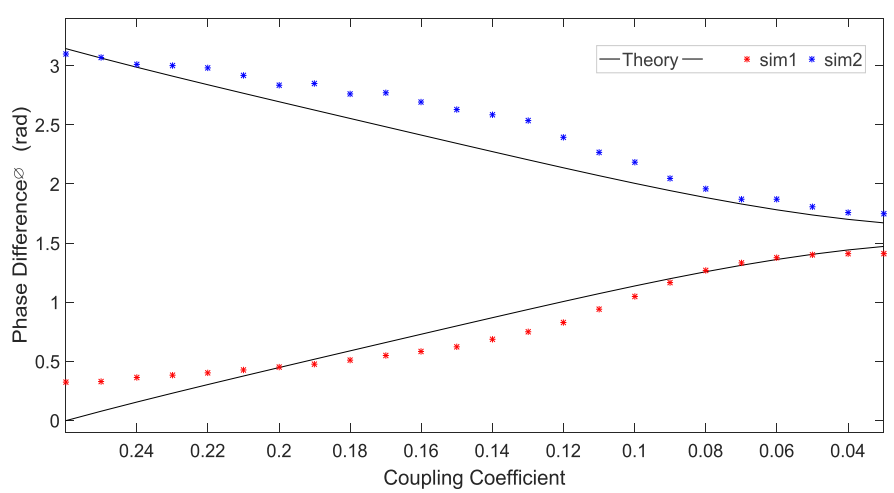

Fig. 8 Phase difference between transmitter and receiver

To compare the characteristics between PT symmetrical WPT system and NPT symmetrical WPT system, a simulation is done which replaces negative resistance at the transmitter with an equivalent positive resistance. The calculation method of transfer efficiency remains unaltered. Fig. 9 presents the comparison between PT symmetric system (blue line) and NPT symmetric system (red line) on transfer efficiency. The transfer efficiency of PT-symmetric system is higher than NPT symmetric system in the whole process and retains relatively stable in the strong coupling region which consistent with the equation (15), conversely, the NPT symmetric system maintains a downward trend with the variation of coupling coefficient. In addition, the resonance frequency and phase difference of the NPT symmetric WPT system keep unaltered.

Fig. 9 Transfer efficiency of PT symmetric system and NPT

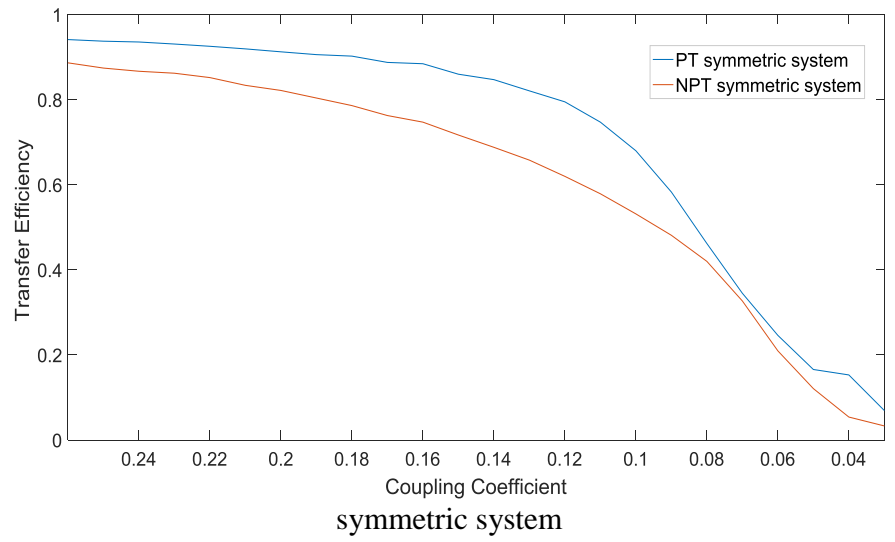

Therefore, the PT symmetrical circuit adjusts the resonance frequency spontaneously in the strong coupling region, causing equal-amplitude oscillations on the transmitter and the receiver. The power transfer efficiency of the system is higher than $80 \%$ and is relatively stable, which is higher than the NPT symmetrical system. The robustness of the PT-symmetric system in strong coupling region could overcome the shortcoming of the transfer efficiency changing with distances. 


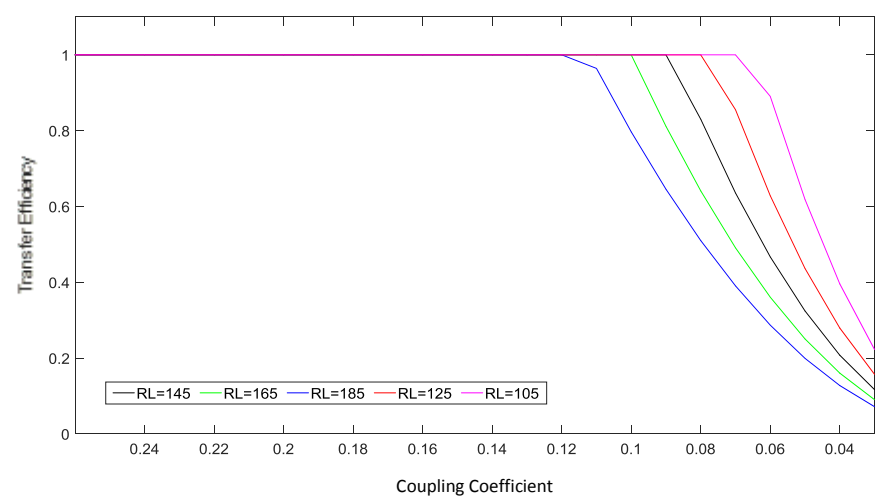

Fig. 10 Theoretical curves of parity-time symmetric circuit with variable resistance

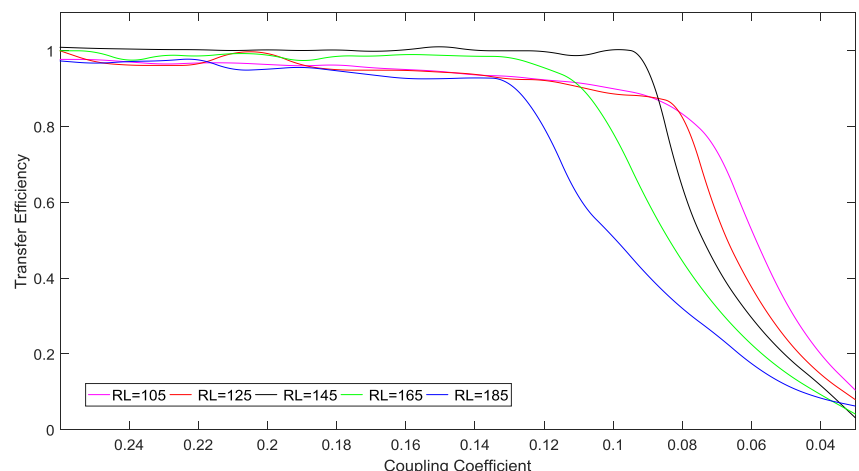

Fig. 11 Simulation curves of parity-time symmetric circuit with variable resistance

A group of simulation with variable load resistances is designed to explore whether the system maintains a stable transmission state with variable circuit parameters. Five sets of simulation with the value of load resistance are $105 \Omega, 125 \Omega$, $145 \Omega, 165 \Omega$, and $185 \Omega$. Figure 10 shows five theoretical efficiency curves corresponding to the different resistance, the five lines of pink, red, black, green and blue represent the efficiency curve corresponding to increasing resistances. Figure 11 shows the simulation result curves. These two sets of curves are roughly in agreement.

\section{CONCLUSION}

This paper analyzes the wireless power transfer system based on the parity-time-symmetry theory. Firstly, the circuit model of the WPT system is established, the relationships of the amplitude and phase difference between the output voltage of the nonlinear gain and the load voltage are found. The characteristics of system resonance frequency with the coupling coefficient are analyzed and the transfer efficiency is obtained. Then making a comparison of the PT-symmetric system with NPT-symmetric system, results indicate that the transfer efficiency of PT-symmetric system has always higher than $80 \%$ in the strong coupling region. When the load resistance changes, the system still maintains efficient and stable transmission within a certain range, indicating that the system still has high robust applicability to the changed coupling coefficient when the circuit parameters are changed. The transmission characteristics of PT-symmetric circuits have been verified through simulations based on PSIM.
The PT-symmetric WPT system is convenient to implement because of its simple circuit structure. The property of automatically adjusting the resonance frequency in the strong coupling region makes this system apply to electric vehicles and mobile phone wireless charging technology easily. At present, the PT-symmetric WPT system has not been applied to the underwater system, the eddy-current loss is the main factor affecting the efficiency, compensation mechanism will be considered in the actual underwater system. The class-D power amplifiers and full-bridge circuits also could be used as system nonlinear gain to achieve variable performance. The future researches could regard the frequency tracking mechanism and multi-coils WPT system.

\section{REFERENCES}

[1] L.H. Wu, B. Zhang. "Overview of Static Wireless Charging Technology for Electric Vehicles: Part I". Transactions of China Electrotechnical Society, vol. 35(6), pp. 1153-1165, 2020.

[2] X.M. Fan, L.L. Gao, X.Y. Mo, Q. Zhao, E.J. Jia. "Overview of Research Status and Application of Wireless Power Transmission Technology". Transactions of China Electrotechnical Society, vol. 34(7), pp. 1353-1380, 2019.

[3] Y. Ra'Di, B. Chowkwale, C. Valagiannopoulos, F. Liu, A. Alu, C.R. Simovski, S.A. Tretyakov. "On-site wireless power generation." IEEE Transactions on Antennas and Propagation, vol. 66(8), pp. 4260-4268, 2018.

[4] A.P. Sample, D.A. Meyer, J.R. Smith. "Analysis, experimental results, and range adaptation of magnetically coupled resonators for wireless power transfer." IEEE Transactions on industrial electronics, vol. 58(2), pp. 544-554, 2011.

[5] A. Kurs, A. Karalis, R. Moffatt, J.D. Joannopoulos, P. Fisher, M. Soljacic. "Wireless power transfer via strongly coupled magnetic resonances." Science, 317(6), pp. 83-86, 2007.

[6] E.B. Balust, A.E. Aroudi, P. Fisher, E. Alarcon. "Unveiling nonlinear dynamics in resonant inductively coupled wireless power transfer." 2014 IEEE International Symposium on Circuits and Systems (ISCAS). IEEE, pp. 2612-2615, 2014.

[7] Y.Y. Liu, H.W. Feng, "Transmission efficiency analysis for magnetic coupled resonance wireless power transfer system in over-coupled region". Journal of Nanjing University of Science and Technology, vol. 43(3), pp. 300-305, 2019.

[8] X.L. Dai, W.Q. Niu, M.X. Cheng. "Analysis on the frequency bifurcation phenomenon of contactless power transfer system", Journal of Power Supply, vol.10, pp. 67-71, 2012.

[9] W.Q. Niu, W. Gu, J.X. Chu, A.D. Shen. "Frequency splitting patterns in wireless power relay transfer", Circuits Devices \& Systems Iet, vol. 8, pp. 561-567, 2016.

[10] W.Q. Niu, J.X. Chu, W. Gu. "Exact Analysis of Frequency Splitting Phenomena of Contactless Power Transfer Systems". IEEE Transactions on Circuits \& Systems I Regular Papers, vol. 60, pp. 1670-1677, 2013.

[11] L.M. Chen, Y.L. Cui, G. B. Yan. "Research on Frequency Tracking Control of Magnetically Coupled Resonant Wireless Power Transmission System", Power Electronics, vol. 11, pp. 3-6, 2014.

[12] I. Shunta, P. W. Yeon, F. Hiroshi. "Wireless power transfer with zero-phase-difference capacitance control", IEEE Transactions on Circuits and Systems I: Regular Papers, vol. 62, pp. 938-947, 2015.

[13] G. Eleni, K. Georgios, M. Stefanos. "Variable frequency controller for inductive power transfer in dynamic conditions". IEEE Transactions on power electronics, vol. 32, pp. 1684-1696, 2016.

[14] D. Shuai, W.Q. Niu, W. Gu. "Lateral Misalignment Tolerant Wireless Power Transfer with a Tumbler Mechanism." IEEE Access, vol. 7, pp. 125091-125100, 2019.

[15] L.Q. Gao, L.R. You, X.Q. Wen. "Frequency tracking technology in magnetically coupled resonant wireless power transmission system", Science Technology and Engineering, vol. 19, pp. 125-131, 2019.

[16] C. Huang, Y.M. Lu. "Frequency tracking detuning control of magnetic resonance wireless power transmission system", Journal of Electrical Engineering, vol. 15, pp. 3102-3111, 2019. 
[17] W.Q. Niu, A. D. Shen, W. Gu, "Reactive power compensation for variable load non-contact power transmission system", Journal of Shanghai Maritime University, vol. 32, pp. 49-53, 2011.

[18] W.Q. Niu, A.D. Shen, W. Gu, J.X. Chu, J.H. Wang. "Reactive power compensation for contactless power transfer systems with variable load". Journal of Shanghai Maritime University, vol. 32(1), pp. 49-53, 2011.

[19] K. YAMAGUCHI, Y. YAMAMOTO, T. HIRATA, I. HODAKA. "Resonance and Efficiency in Wireless Power Transfer System". WSEAS Transactions on Circuits \& Systems, vol.13, pp.218-223, 2014.

[20] S.Y. Xie, Y.G. Su, C.S. Tang, L. Chen. "Research on Meander-type Coupled Structure of Capacitively Coupled Power Transfer System". WSEAS Transactions on Circuits \& Systems, vol.14, pp.247-252, 2015.

[21] J. Schindler, A. Li, M. C. Zheng, "Experimental study of active LRC circuits with PT symmetries". Physical Review.2011, A84,040101(R).

[22] J. Schindler, Z. Lin, J. M. Lee. "PT-symmetric electronics". Journal Physics A: Mathematical and Theoretical.vol. 45, 2012

[23] S. Assawaworrarit, X.F. Yu, S.H. Fan. "Robust wireless power transfer using a nonlinear parity-time-symmetric circuit". Nature. vol. 546, pp. 387-390, 2017.

[24] Z.Y. Zhao, "Circuit Model and Analysis of Wireless Power Transfer System Based on Parity Time Symmetry", South China University of Technology, Guangzhou, ON, China, 2018.

[25] W.J. Dong, H. Zhang, C.S. Li, X. Liao. "Research on Variable Gap Wireless Energy Transmission Method for Fuzes Based on Nonlinear PT Symmetry Principle", Acta Armamentarii, vol. 40, pp. 35-41, 2019.

[26] W.J. Dong, H. Zhang, C.S. Li, X. Liao. "Research on Variable Gap Wireless Energy Transmission Method for Fuzes Based on Nonlinear PT Symmetry Principle", Acta Armamentarii, vol. 40, pp. 35-41, 2019.

[27] J.L. Zhou, B. Zhang, W.J. Xiao, D.Y. Qiu, Y.F. Chen, "Nonlinear parity-time-symmetric model for constant efficiency wireless power transfer: Application to a drone-in-flight wireless charging platform", IEEE Transactions on Industrial Electronics, vol. 66, 4097-4107, 2018.

[28] W.J. Dong, C.S. Li, Z. He, L.B. Ding. "Wireless power transfer based on current non-linear PT-symmetry principle." IET Power Electronics, vol. 12(7), pp. 1783-1791, 2019.

[29] M. Sakhdari, M. Hajizadegan, Y. Li, M.C. Cheng, J.C.H. Hung, P.Y. Chen, "Ultrasensitive, parity-time-symmetric wireless reactive and resistive sensors". IEEE Sensors Journal, vol. 18, pp. 9548-9555, 2018.

[30] O. Abdelatty, X.Y. Wang, A. Mortazawi, "Position-Insensitive Wireless Power Transfer Based on Nonlinear Resonant Circuits", IEEE Transactions on Microwave Theory and Techniques, vol. 67, pp. 3844-3855, 2019.
[31] Y.T. Hou, M.S. Lin, W.J. Chen, X. Yang. "Parity-time-symmetric Wireless Power Transfer System Using Switch-mode Nonlinear Gain Element". 2018 IEEE International Power Electronics and Application Conference and Exposition (PEAC). IEEE, vol.14, pp. 1-5, 2018.

[32] X.J. Shu, B. Zhang. "Energy model and characteristic analysis of inductively coupled wireless energy transmission system". Automation of Electric Power Systems, vol. 41(2), pp. 28-32, 2017.

Yu Jin received the B.E. degree from Shandong University of Science and Technology, Qingdao, China, in 2017, the M.E. degree from Institution of Lisbon University, Lisbon, Portugal, in 2019.

She is currently pursuing the M.S. degree with Shanghai Maritime University, Shanghai, China. Her research interests include wireless power transfer and IoT technology.

Wangqiang Niu received the B.E. degree from Xi'an Aerotechnical College, Xi'an, China, in 1998, the M.E. degree from Northwestern Polytechnical University, Xi'an, in 2004, and the Ph.D. degree from Shanghai Jiao Tong University, Shanghai, China, in 2008.

From 2013 to 2014, he was a Visiting Lecturer with McMaster University, Hamilton, ON, Canada. Since 2008, he has been a Lecturer with Shanghai Maritime University, Shanghai, where he has been an Associate Professor, since 2017. His research interests include wireless power transfer, control of marine equipment and prognostics and health management.

Wei Gu received the B.E. and Ph.D. degrees from Shanghai Maritime University, Shanghai, China, in 1982 and 2008, respectively.

Since 1982, he has been a Teacher with Shanghai Maritime University, where he has been a Professor, since 1997, and is currently the Director of the Key Laboratory of Transport Industry of Marine Technology and Control Engineering. His research interest includes marine information control technology.

\section{Creative Commons Attribution License 4.0 (Attribution 4.0 International, CC BY 4.0)}

This article is published under the terms of the Creative Commons Attribution License 4.0 https://creativecommons.org/licenses/by/4.0/deed.en US 